\title{
A PORTABLE STRAIN METER WITH CONTINUOUS INTERFEROMETRIC CALIBRATION
}

\author{
By J. L. Blayney and Ralph Gilman
}

\begin{abstract}
A strain meter has been designed embodying techniques not used in earlier models. Minimum use of heavy and permanent auxiliary fixtures has made possible a portable instrument in which no components need be abandoned in order to change location. Provision for frequent or continuous interferometric calibration, and a photographic record thereof, has provided uninterrupted strain measurements with long-term dependability. Also included is the usual continuous low-speed ink recorder for tidal and secular variations, and a highgain band-pass recorder for the observation of long period waves and free oscillations of the earth. Details are given describing the method used for interpreting the interferometer image and its conversion to strain measurement.
\end{abstract}

\section{INTRODUCTION}

Since a simplified design and a greatly reduced cost of a linear strain meter (Benioff, 1959, 1963) would make possible the proliferation of such instruments over many active regions, the Seismological Laboratory of the California Institute of Technology has placed in operation a strain-measuring instrument of semi-portable design. Through the generous cooperation of the U. S. Coast and Geodetic Survey, space was made available for this installation in the Kipapa tunnel, site of the World Wide Standard Seismograph Station in Hawaii. This tunnel, driven into the side of Kipapa Gulch 100 feet below the rim, is located on the island of Oahu in a region of weathered basalt. The strain tube is 24.4 meters long, in a direction $61.1^{\circ}$ west of north and is placed toward the far end of the 220 foot tunnel. In addition to observations of long waves and free oscillations, measurements of mid-oceanic earth tides and a possible correlation between secular strain on Oahu and movements of the magma beneath the island of Hawaii will be of particular interest.

Although the fused quartz tube is relatively long and is located in a dry tunnel, features which do not lend themselves to portability nor to an arbitrary choice of sites, the design was planned along lines anticipating a possible shortening of the tube and eventual elimination of the tunnel requirement. Alternate locations may include the basement of an existing building, or a shallow excavation covered by an insulating layer of earth.

\section{Strain RoD}

One important change from earlier designs is in the technique for suspending the quartz tube. Rather than hanging the tubing with stainless steel wire from the walls of a concrete channel, a double-hinged support anchored to the floor directly beneath the quartz contains a self-locking clamp to hold the quartz tube firmly to the upper part of the hinge. This inverted pendulum approach was first proposed in 1951 by F. E. Lehner of the Seismological Laboratory staff. Figure 1 shows the hinge and 
clamp structure carrying a short sample of the quartz tubing. Each ten-foot length of the tubing is carried by two of these supports spaced five feet apart, the thickness of the hinges and the resulting restoring force being such that the system becomes an inverted pendulum approaching instability. Thus, when the lengths are cemented,

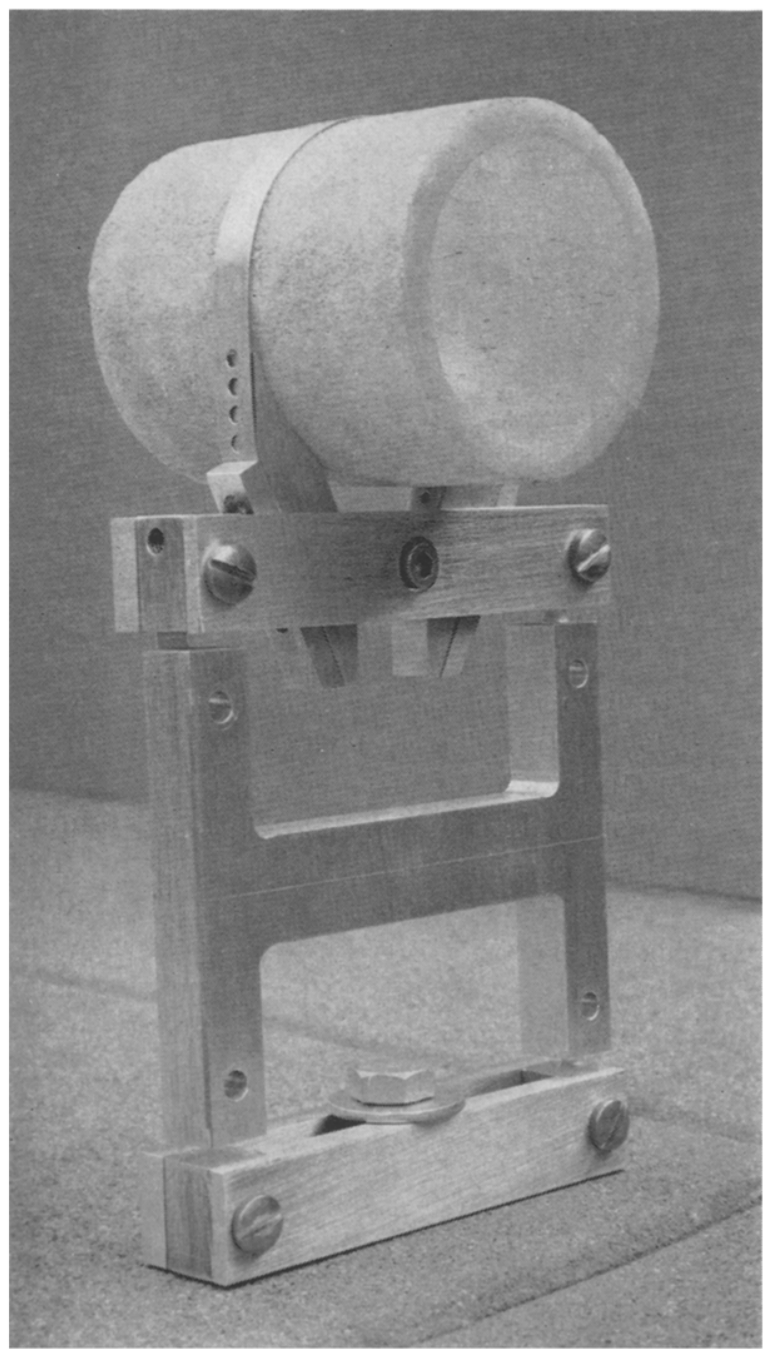

FIg. 1. Hinge and clamp strueture earrying sample of quartz tubing. Weight of quartz produces pinching action of flexible strap by forcing wedge-shaped terminations against adjusting screws. Position of quartz may be shifted vertically or laterally by these screws before final clamping (single bolt, upper center).

as described by Benioff (1959), and the unit is fastened rigidly to the rear pier, the entire length of tubing will be laterally restrained but should be practically free of axial stresses. Hinge dimensions to meet the required restoring force approach the maximum allowable slenderness ratio for the material used, that is, where an in- 
creased load would result in failure of the hinge by buckling and compression simultaneously.

The terminating piers are commercial precision-ground cast-iron machinists' surface plates of four-inch-thick ribbed construction, cadmium-plated for corrosion resistance. These were filled with concrete mortar and cemented into excavations one inch deep in the concrete floor of the tunnel, thus keeping the volume of fresh concrete at a minimum. The plate was leveled and allowed to settle under its own weight into the setting concrete. Here is a somewhat radical divergence from former

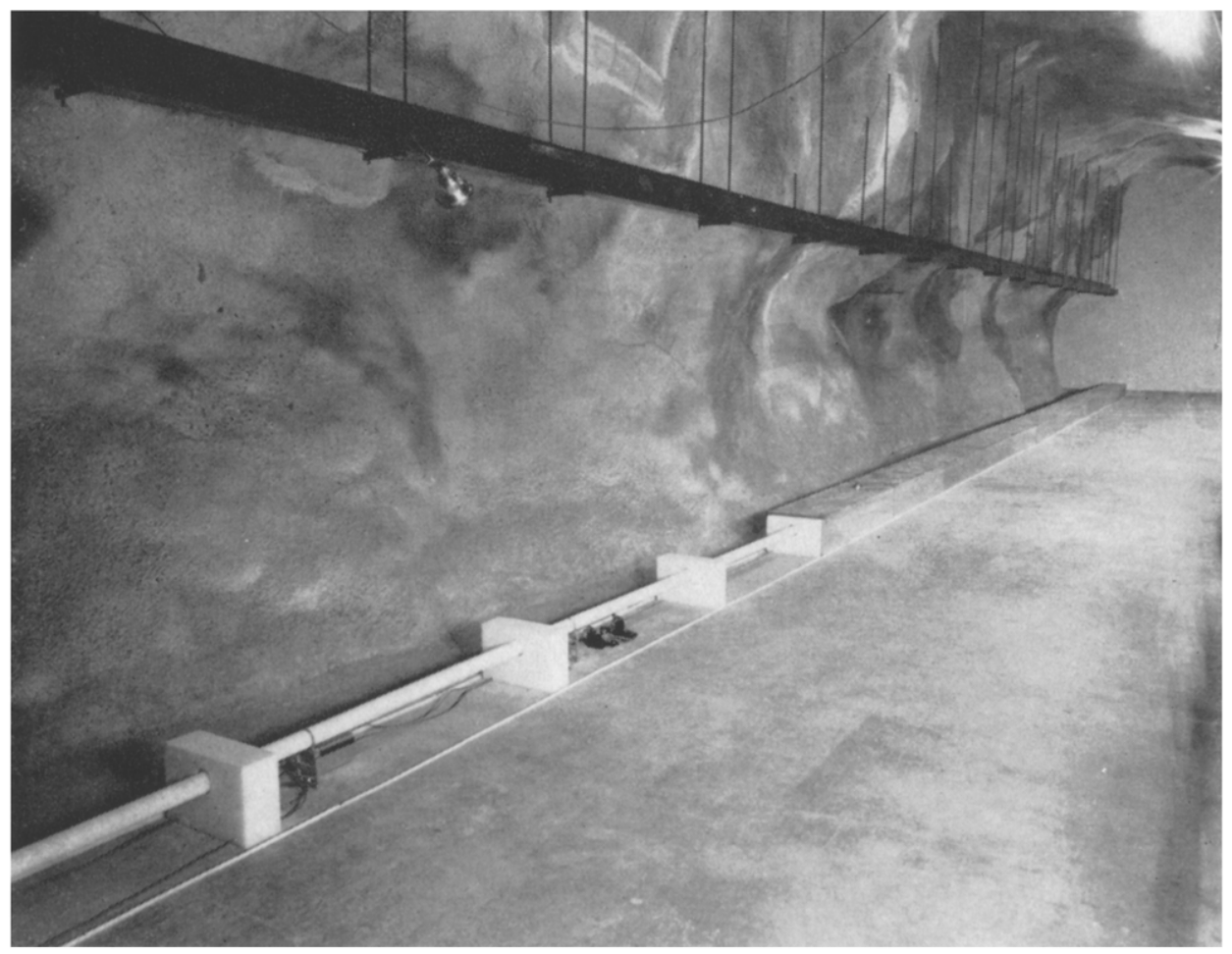

FIG. 2. General view of Kipapa strain meter, excluding free end termination and pier. Device at center, below quartz, is test signal apparatus (see text).

practice whereby a pipe, twelve inches in diameter and six feet long, was cemented into a five-foot-diameter hole. The large volume of concrete involved, as well as the serious disturbance of the surrounding rock due to blasting, resulted in a lengthy settling period before tidal strain measurements could be made.

In the Kipapa installation it is intended to show that a concrete slab will bond rock fractured during tunnel construction, and thereby serve as an accurate coupling for the transfer of strain from the undisturbed rock below.

The entire length of the instrument, including the terminating piers, is protected by a wooden cover measuring ten by sixteen inches and divided into convenient five-foot lengths for easy removal (Figure 2). These sections are positioned 
and kept in place by five-inch thick, rigid foam, polystyrene dividers, which were cemented to the floor at the time the quartz was supended. Three-inch diameter holes through the polystyrene allow ample clearance for the two and one-half inch quartz tubing, but are sufficiently small to prevent excessive convection between sections. A final seal between the cover boxes and the concrete floor is provided by a wedge of flexible foam polyethylene.

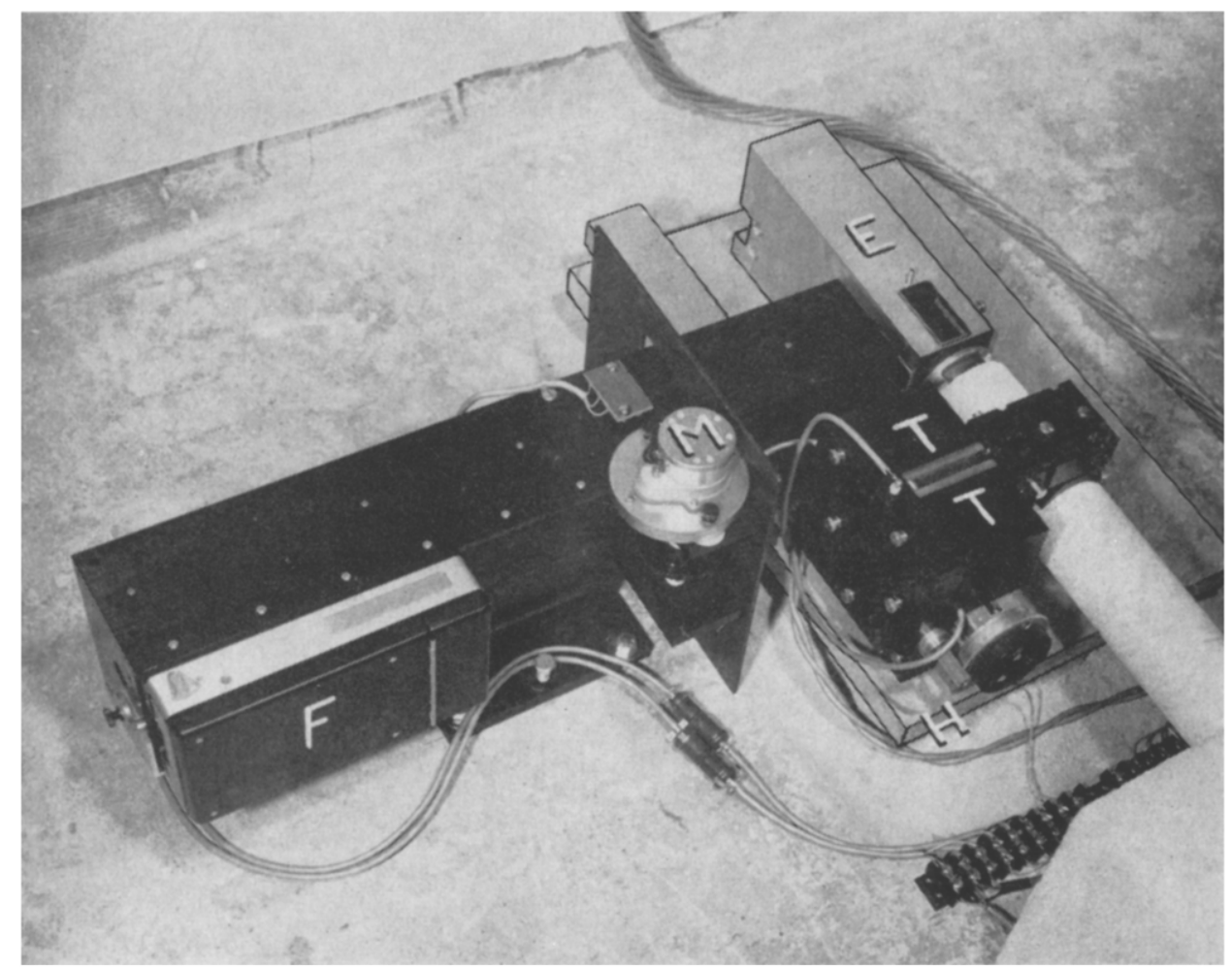

FIG. 3. Free end termination showing transducer assembly and interferometer camera. F-Polaroid film-pack adapter. M-Continuous drive motor for lateral progression of filmpack unit. E-Interferometer housing and pier block. T-Resonant circuit box. H-Centering motor and micrometer drive unit.

\section{TransduCER}

Two devices are installed at the free end of the tube (Figure 3). One measures the relative movement between the quartz and the pier by use of a Fabry-Perot interferometer permanently mounted in line with the quartz axis. The purpose of the interferometer is to provide a stable and frequent first order calibration of the electrical sensing device, in addition to maintaining a dependable reference zero for long-term strain measurements. Since the interferometer plates are co-axial with the quartz tube, any indicated change in separation must correspond to earth strain, assuming no internal or external stress changes on the quartz, piers, or associated parts. The other device measures the displacement of a conductive plate 
mounted on and to one side of the quartz, a few inches back from the free end. The capacitances formed by two conductive surfaces, spaced about one millimeter on each side of this plate, become the capacitive portions of two series-resonant circuits, which are excited by a transistorized five-megacycle crystal oscillator. The potentials developed across the inductors of these resonant circuits are rectified by low-capacitance, high back-resistance germanium diodes, and the resulting voltages added in opposition to give zero volts output at balance (Benioff, 1959). Each inductor and its associated diode is housed in a two-and-one-half-inch cubical aluminum-shielded box. One face of the box is a one-quarter-inch thick, copper-clad, epoxy-glass panel upon which the capacitor plate is etched and gold-plated. This is done in a manner which almost completely shields the coil and yet keeps the stray capacitance at a minimum. The moving element contributes about two picofarads to each resonant circuit, which is about one-quarter of the total tuning capacitance. Either box can be removed by the loosening of two thumbscrews, in case of failure of the enclosed diode, and a new unit can be placed accurately enough to require a minimum of retuning. Sensitivity of the transducer is nominally 0.25 volts per micron when a convenient working gap of about one millimeter is used. Output impedance is quite high and, in other installations, is transformed to a few hundred ohms by a complementary emitter-follower circuit before being carried by shielded cable to various recorder combinations. In the Oahu instrument, however, no follower circuit is used. The signal is divided by a factor of 100 , passed through a filter designed to suppress short-period microseisms, then fed into an ink-writing recorder having one megohm input impedance and a full scale sensitivity of ten millivolts (Leeds and Northrup Speedomax G).

Using a working gap of one millimeter, this arrangement yields a D.C. magnification of 93,000 ; that is, one micron displacement of the quartz tube, relative to the transducer pier, will result in a deflection of 93 millimeters on the paper record. Expressed in terms of strain sensitivity, a record deflection of one millimeter is equivalent to ground strain of $4.41 \times 10^{-10}$.

A second recorder, having identical input characteristics, is operated through a filter designed to attenuate tidal and microseismic frequencies. This band-pass filter, with peak response at one hour, is similar to the one used at Naña, Peru, and discussed by Benioff (1963); however, unusually high microseismic noise at Kipapa has made additional attenuation at short periods necessary. The time scales on the direct and filtered records are, at present, nine inches per day and six inches per hour respectively, with time marks each six hours on the former and each minute on the latter. Figure 4 shows a sample trace from each recorder.

Keeping the trace within the range of the direct recorder is accomplished by a remotely controlled motor which moves the precision worm-drive of a transverse micrometer unit (Gaertner M 340). This unit carries the resonant circuit boxes and moves them along the strain axis until the sensing plate is centered between them. The vernier scale on the readout drum can be read to a half-micron accuracy, and these accumulative readings are compared with the interferometric plot. The corresponding displacements of the recorded trace, whenever the attendant centers the system, provide an occasional check on the transducer gain. These manipulations, however, have no effect upon the interference pattern of the interferometer, 
whose parts were designed to have no possible means of adjustment other than the parallelism control of the interferometer plates, and this has only a second-order effect upon the plate spacing.

A second remotely controlled device initiates test signals used in calibrating the capacity transducer recorders. Small cam-actuated springs extend or compress the quartz tube, producing a simulated signal. One spring produces a sinusoidal variation of a one hour period and is opposed by another set arranged to introduce step pulses in either direction. Cam throw may be adjusted to modify signal amplitude

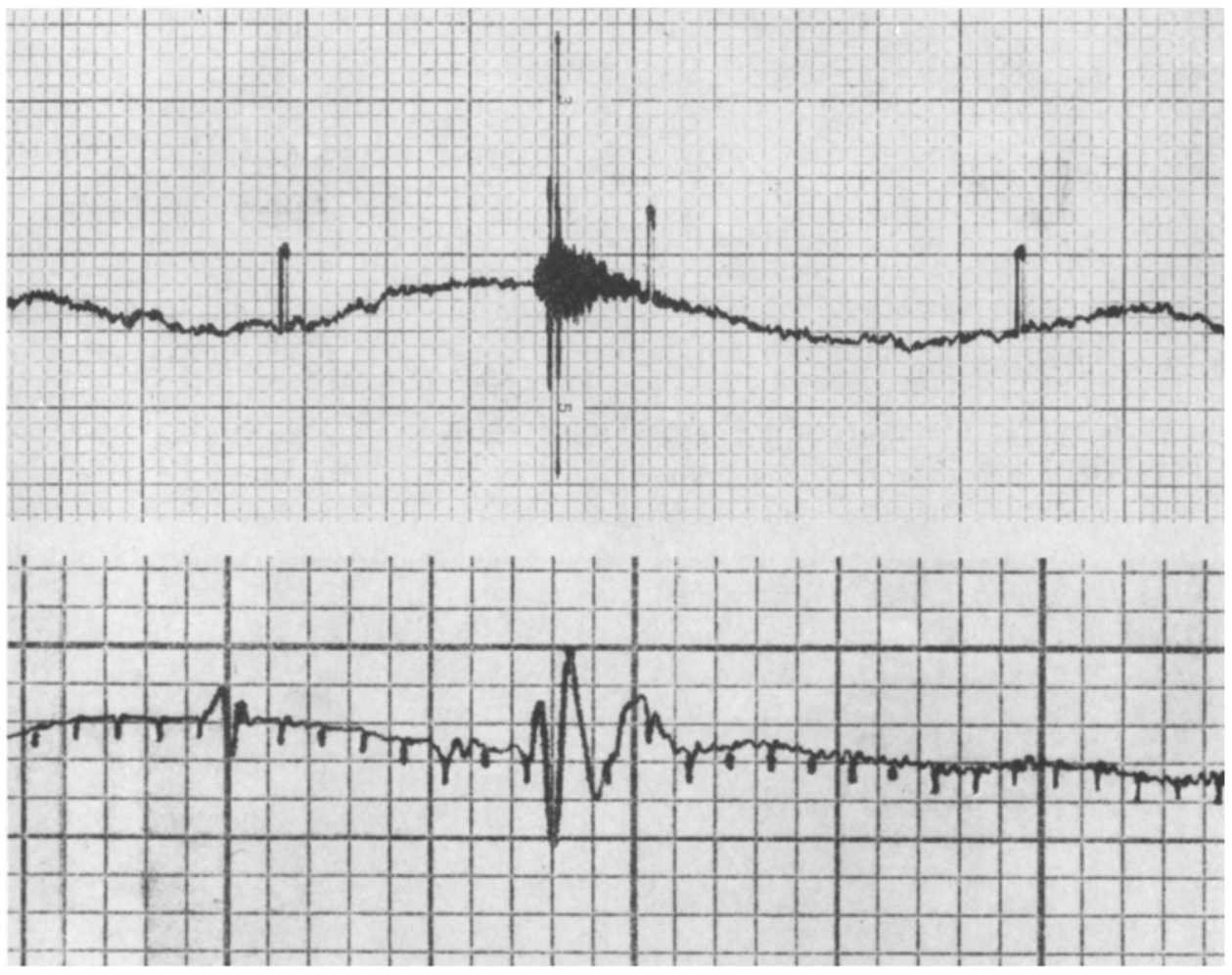

Fig. 4. Upper trace: 20-hour sample from direct recorder showing earthquake of 16 June 1964; origin time 04:01:44.3. Lower trace: 30-minute sample from filtered recorder showing $S$ and $R_{1}$ arrivals from: same earthquake.

of the sine wave, but amplitude of the step pulses is determined by limit stops in the system and the choice of spring rates. Simultaneous recording of the test signals by the interferometer camera and the transducer recorders provides an accurate and convenient method for determining sensitivity.

In order to make a more meaningful comparison with pendulum seismometers, curves have been constructed showing frequency response in terms of ground displacement. The response of a strain meter to ground displacement is inversely proportional to wavelength, when the strain meter is relatively short. Love and Ray- 


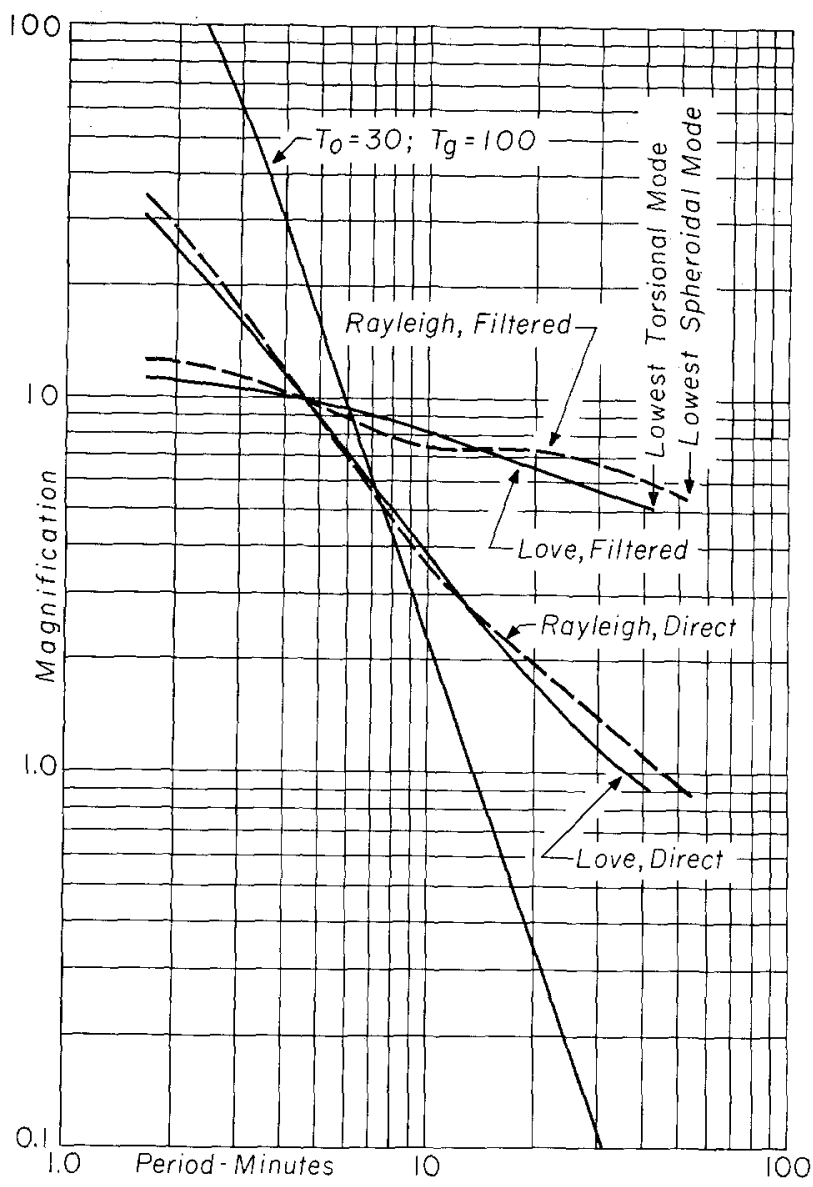

Fic. 5. Response curves showing magnification of ground displacement versus ground period for Kipapa long-period pendulum seismograph and strain meter.

TABLE 1

Phase Velocities

\begin{tabular}{r|r|r|r}
\hline \multicolumn{2}{c|}{ Love Waves } & \multicolumn{2}{|c}{ Rayleigh Waves } \\
\hline $\mathrm{T}, \mathrm{sec}$ & $\mathrm{C}, \mathrm{km} / \mathrm{sec}$ & $\mathrm{T}, \mathrm{sec}$ & $\mathrm{C}, \mathrm{km} / \mathrm{sec}$ \\
\hline 100 & 4.65 & 100 & 4.12 \\
150 & 4.78 & 150 & 4.33 \\
250 & 5.08 & 250 & 4.96 \\
400 & 5.56 & 400 & 5.93 \\
600 & 6.12 & 600 & 6.62 \\
1000 & 6.77 & 707 & 6.68 \\
1300 & 6.40 & 1000 & 6.35 \\
1500 & 6.88 & 1500 & 5.83 \\
2500 & 6.30 & 3228 & 5.06 \\
\hline
\end{tabular}


leigh wave phase velocities were used to determine the appropriate wavelength associated with each period. This somewhat arbitrary choice was influenced by the wide interest in these waves at ultra-long periods. The values for phase velocity, supplied by Don L. Anderson (personal communication) have been averaged from curves summarizing recent observations (Table 1).

Figure 5 shows the response of the Kipapa pendulum seismograph $\left(T_{0}=30\right.$; $T g=100)$ together with the Rayleigh and Love wave response of the strain meter. For observing ultra-long-period surface waves, free modes and secular strains in the earth, the usefulness of the strain meter is demonstrated.

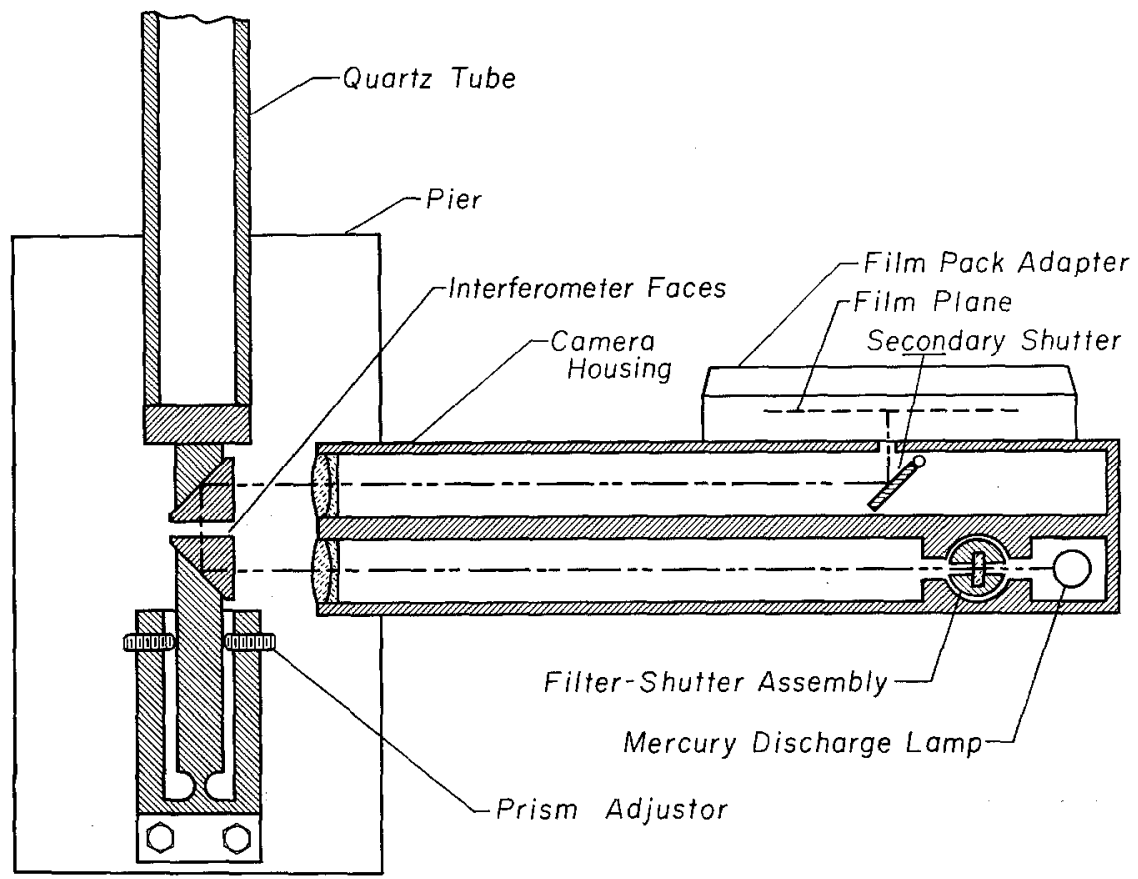

Frc. 6. Schematic diagram showing optical path through interferometer from light source to film plane. Shutters are shown in exposure position.

\section{INTERFEROMETER}

The interferometer unit, although used previously as a calibrator, has become a permanent and automatically recording part of the strain instrument (Figure 6). The interferometer plates are in the form of right-angled isosceles prisms with oneinch faces; flat to within one-tenth wavelength, silvered to the extent of five per cent transmission, and overcoated for protection of the silver. One prism is fixed to the end of the quartz tube, on axis, and the other, about two millimeters from it, is housed in a block mounted on the pier and provided with means for adjusting its parallelism relative to the free prism. Both prisms are isolated from the atmosphere in a desiccated compartment, sealed to the moving element with a ring of silicone grease. The camera contains a mercury discharge tube and a multiple filter designed to select the spectral frequencies to be passed through the interferometer 
and photographed by an incorporated Polaroid film-pack unit. The entire assembly is mounted rigidly to the concrete floor adjacent to but not touching the pier (Figure 3). This was done to provide some mechanical de-coupling from vibrations of the camera motors. The camera and light and source lenses, each sixteen inches focal length, are exposed to the interferometer through an optical window in the prism compartment. Exposure occurs once a day and proceeds in a manner designed to minimize the total light energy falling upon the interferometer parts and the amount of stray light seen by the high-speed film (ASA 3000). This is done by the actuating motor in the following fifteen-second sequence. After the mercury lamp is turned on,

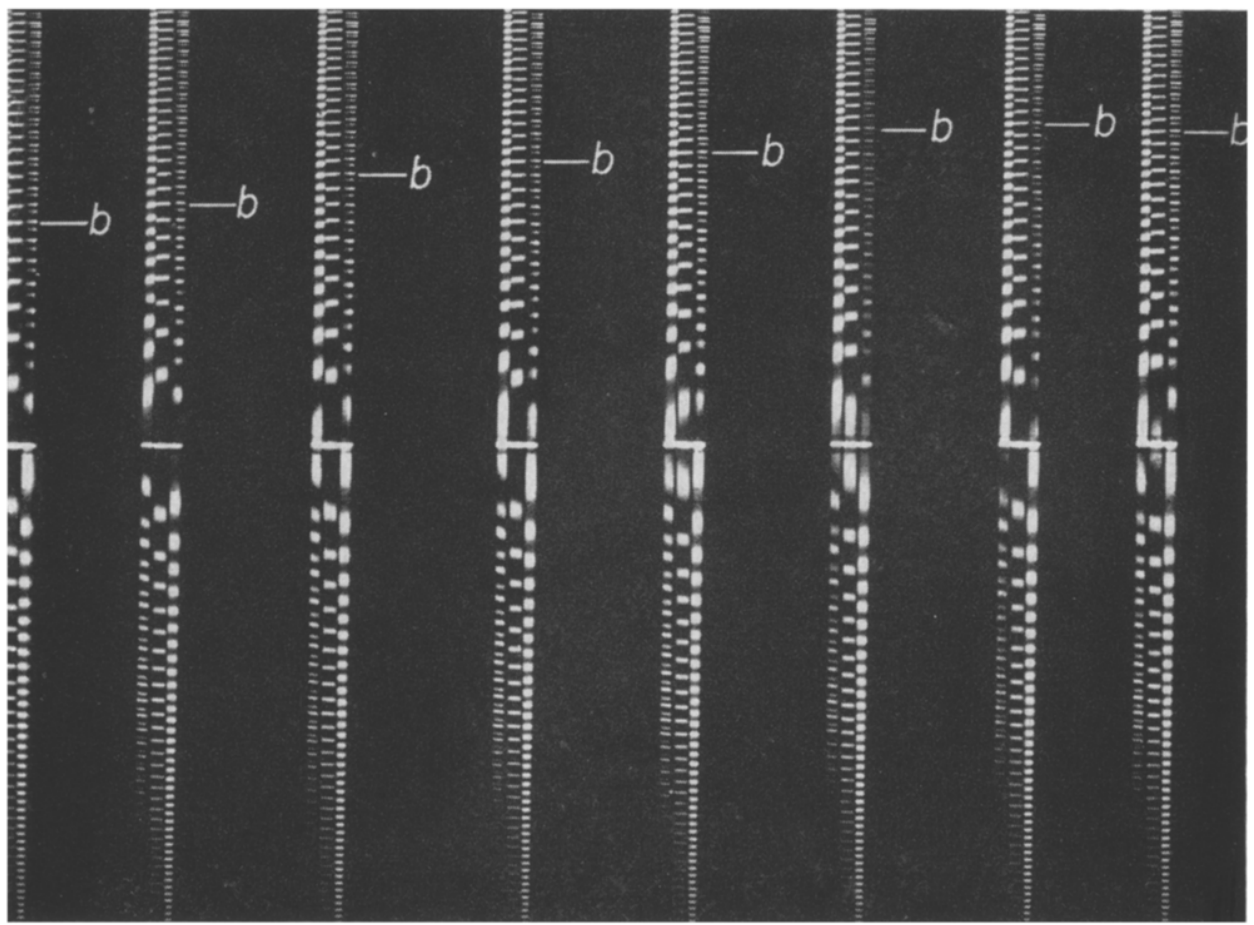

FIG. 7. Reproduction of Polaroid print showing eight daily exposures through interferometer. Line designated $b$ is easily identifiable blue-green-yellow coincidence. (See Figure 8.)

the opening to the film pack is uncovered and directed by a mirror toward the interferometer. The rotary shutter then passes the light source across the composite strip filter, sending the filtered light through the interferometer to form an image of the filter at the film plane. After a two second exposure, the film pack is again covered, and the light turned off. By use of a continuously running motor, the film pack holder is progressed laterally at the rate of one-half inch per day, so that each print displays a series of eight images representing one week of daily samples (Figure 7). The first exposure is taken a few minutes after the last one of the preceding week. This procedure is helpful in maintaining chronological continuity of the prints. 


\section{Fringe Measurement}

At this point a description of the interferometer image is in order. Figure 8 is a photograph showing the upper half of one of the daily samples. The complete image

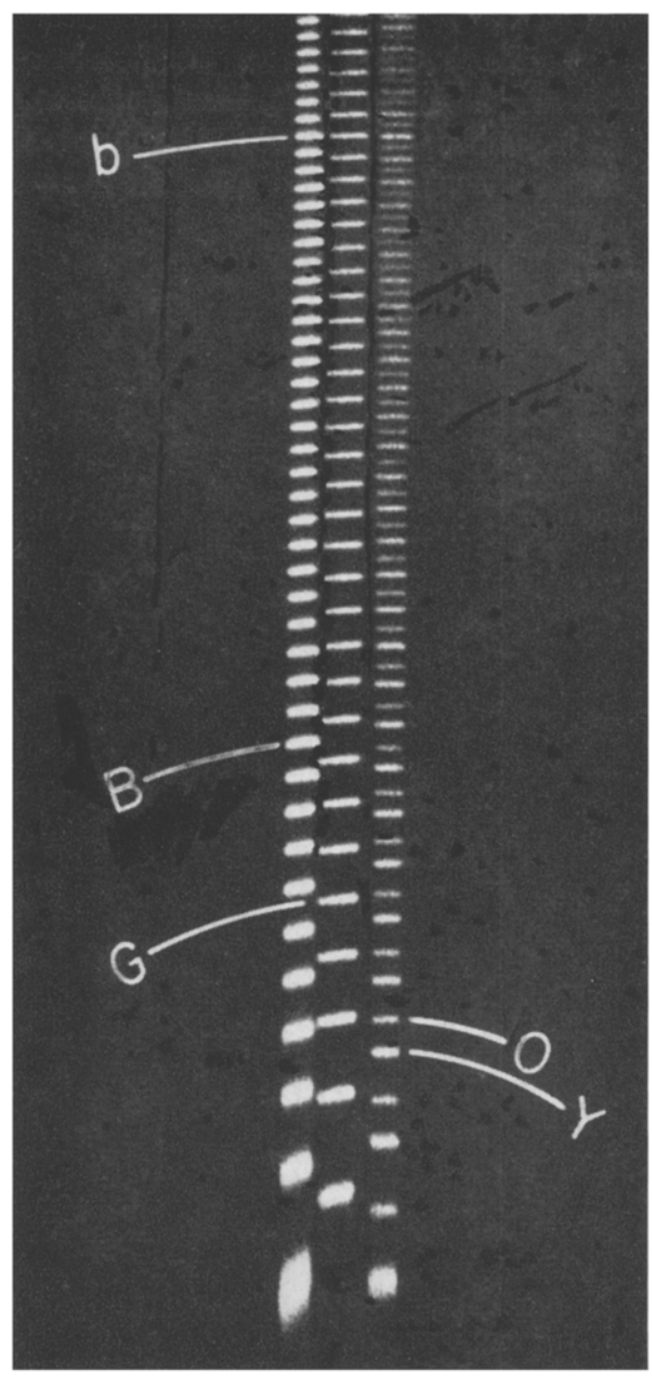

FIG. 8. Detailed view showing upper half of interferometric image. Comparison of mercury green line pattern (center strip) with adjacent patterns for blue, yellow, and orange, shows identifying coincidences and their trends.

is a radial section, three millimeters wide by 73 millimeters long through the series of concentric circular fringes, whose spacings are proportional to the square root of consecutive integers. The strip is further divided into three sections exposed through appropriate filters for the mercury green line $(5460.7348 \AA)$, the blue line (4358.3277 $\AA$ ), and the yellow and orange lines $(5769.5982 \AA$ and $5790.6630 \AA$, 
respectively). The difference in the squares of the diameters of any two fringes of order $N_{1}$ and $N_{2}$ respectively is given by

$$
D_{1}{ }^{2}-D_{2}{ }^{2}=\frac{4 f^{2} \lambda\left(N_{2}-N_{1}\right)}{d},
$$

where $D_{1}$ and $D_{2}$ are the diameters of the fringes of orders $N_{1}$ and $N_{2}$, respectively, since the order of interference is highest at the center of the circular pattern.

Let

$$
\begin{aligned}
& f=\text { the focal length of the imaging lens } \\
& \lambda=\text { the wavelength of the light } \\
& d=\text { the separation of the interferometer plates; }
\end{aligned}
$$

then:

$$
d=\frac{4 f^{2} \lambda N}{D_{1}^{2}-D_{2}^{2}}
$$

This formula allows the plate separation $d$ to be determined to the first order of accuracy by measuring the diameters of any two fringes separated by $N$ orders. The per cent error in this determination of $d$ will be about twice the per cent error in the measurement of the fringe diameters. Since the fringes in this type of interferometer can be extremely sharp (sharpness increases with the coefficient of reflectivity of the surfaces), the apparent width of a fringe being about one-twentieth or less of the fringe spacing, a diameter measurement accurate to within a few per cent is easily attained. In making use of the composite image, all measurements are in terms of the order number of the green line. By treating the interference pattern of each wavelength as a scale with imaginary decimal subdivisions, tables have been prepared showing the fractional-order number of the green line for which each of the other wavelengths becomes theoretically coincident. In this sense, coincidence of fringes of light from two spectral lines of wavelengths $\lambda_{1}, \lambda_{2}$, occurs when

$$
N \lambda_{1}=(N+M) \lambda_{2}
$$

where $M$ is an integer, negative if $\lambda_{2}>\lambda_{1}$, and $N$ is in general not an integer, because of the incommensurability of $\lambda_{1}$ and $\lambda_{2}$.

On rearranging

$$
N=M \frac{\lambda_{1}}{\lambda_{1}-\lambda_{2}}, \quad M=1,2,3,4, \cdots
$$

In Table $2, \lambda_{1}$ becomes the wavelength of the green line, $5460.7348 \AA$ and $\lambda_{2}$, the wavelength of the comparison line. The numbers given are the successive values of $N$ for coincidence of the green fringes with the blue, orange, and yellow fringes, respectively, for successive values of $M$. The actual fringes on each side of these 
TABLE 2

Coincidences for Green Line Orders

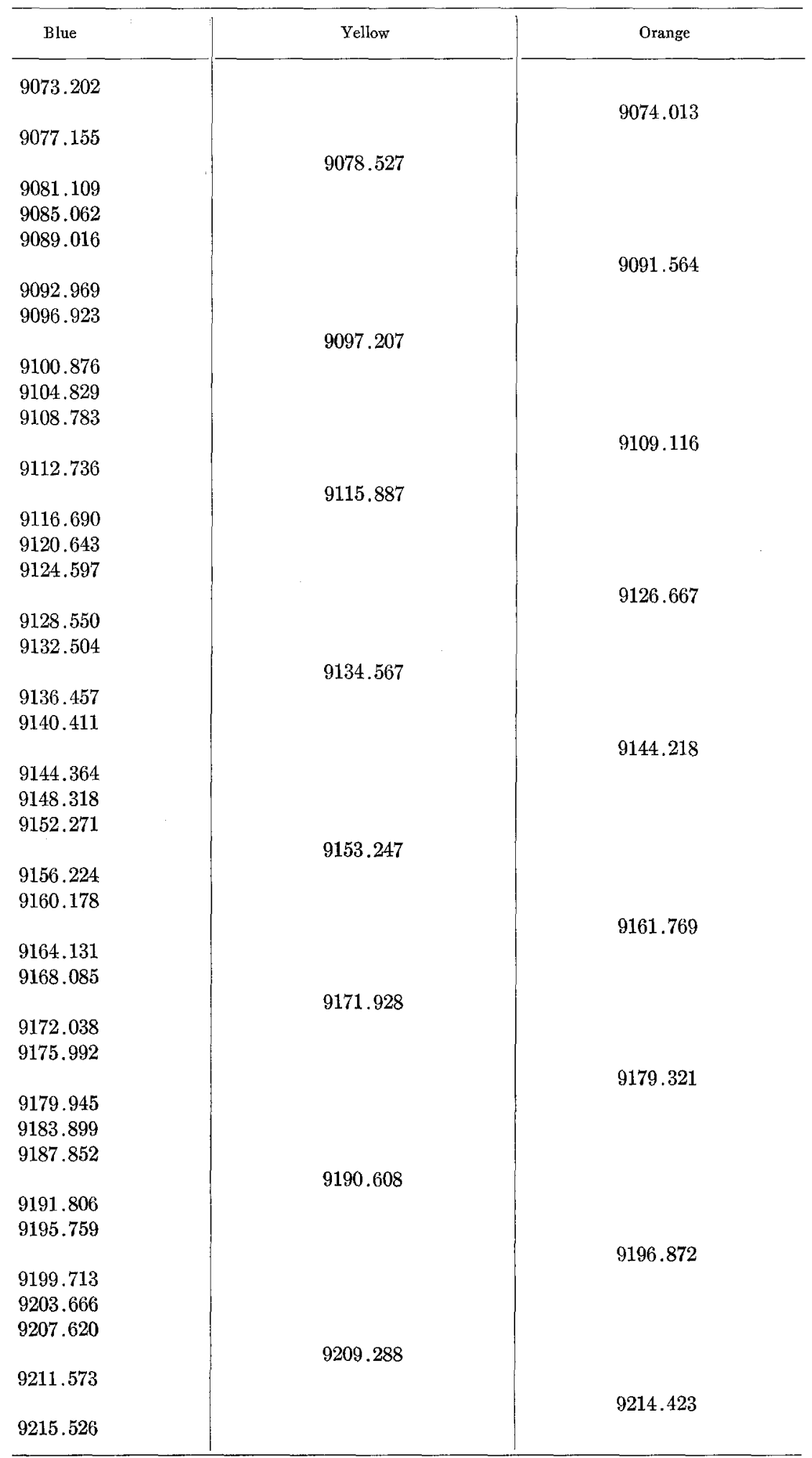


values will show an approximate coincidence, and the fractional value itself can be thought of as the point at which the longer wavelength pattern catches up with and passes the one of shorter wavelength as the order number increases, that is, as the interferometer plates are moved apart. As can be seen from a study of Figure 7, Figure 8, and Table 2, coincidence between the green and blue fringes occurs approximately every four orders of the green line. Coincidences of the green with the yellow and orange occur about every 19 and 18 green orders respectively. The yellow and orange fringes alternately split and pair approximately every 291 green orders. The yellow and orange lines, which differ by only 21 angstroms, and hence are passed by the same filter, appear together in the same strip of the photographic image. They can be separately identified, however, since the shorter wavelength line is considerably brighter.

The process of determining the exact separation of the interferometer plates for any given photographic sample is first to obtain the approximate value by use of equation (2). A five per cent accuracy in this determination would narrow the range of the possible order of interference to about 200 fringes in the tables. The next procedure is to pick a convenient green-yellow coincidence in the photograph and count the number of green orders down (larger diameters) to the adjacent green-orange coincidence. This number, together with the number of orders up (smaller diameters) to a similar green-orange coincidence, gives a ratio which indicates the degree of splitting or pairing of the yellow and orange lines. Since this ratio recurs only every 291 fringes, the green-yellow coincidence and its order number have been tentatively identified in the tables. Without reference to the blue fringes, however, there is still some ambiguity, since there is a possible observational error in the identification of a green-yellow or a green-orange coincidence by plus or minus one fringe, and the separation of adjacent similar pairs of coincidences changes by about the same amount. A green-blue coincidence, on the other hand, is easily identified because the next green order, either up or down, shows a discontinuity with the blue by twenty per cent. A further check for a correlation between the photograph and the tables, as regards the blue-green coincidences, will lend positive identification to the fringe orders. An additional useful identifying occurrence is the shift from an exact blue-green coincidence to a split condition, where two blue fringes are bracketed by two green fringes with a ten per cent discontinuity. This occurs approximately every 95 green orders. The actual order at the center of the pattern, and hence the exact number of wavelengths in the separation of the interferometer plates, can be determined by merely counting orders to the pattern center from any identified fringe. The measurement of the last fractional part of the central order is made using a scale especially constructed for the particular region of order numbers involved. The division must fit the region since the coarseness of the pattern varies with plate separation. Construction of the scale is based on the fact that for any given interferometer plate separation which is an integral number of half-wavelengths, $N$, the linear differences in diameters along the photographic image will be constant and unique for the fringe orders $N$ $-M^{2}, M=0,1,2,3, \cdots$, that is, for the orders $N,(N-1),(N-4),(N-9)$, etc., as shown in Figure 9 . With this radial difference taken as unity, the space between $(N-1)$ and $(N-2)$ is then subdivided into ten divisions corresponding 
to the values $(N-1.1),(N-1.2), \cdots,(N-2.0)$. These are spaced in terms of the above-defined unit by the values $\sqrt{1.1}, \sqrt{1.2} \cdots \sqrt{2.0}$, with the result that the scale appears almost linear. It is this fact that determined the choice of the interval $(N-1)$ to $(N-2)$. While the interval $(N-0)$ to $(N-1)$ is about two and one-half times larger, its extreme non-linearity, and the resulting non-symmetry of a fringe about its mid-point in this region, would increase the error in measuring fringe diameters. The scale is expanded by projection along an eightdegree line and the divisions and decimal numerals scribed on the underside of a lucite plate. When placed in contact with the interferometric photograph, the scale becomes visible as a white line against the black background, but as a dark line over

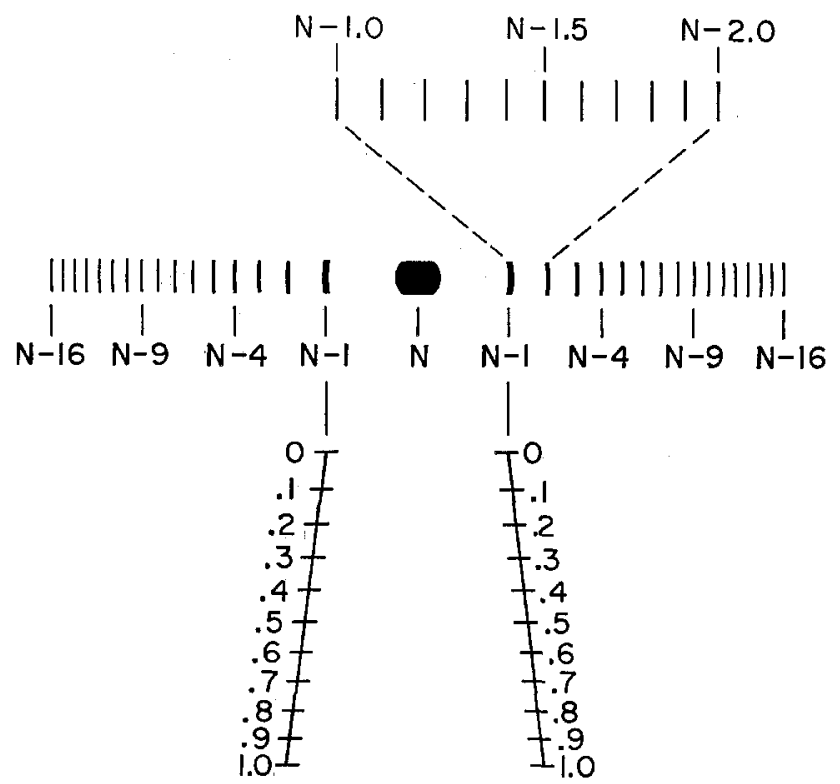

FIG. 9. Horizontal distances between decimal subdivisions shown above represent scale used in determining fractional order at center of interference pattern. As central order increases from $N$ to $N+1$, diameter of fringe $N-1$ changes from top dimension of trapezoidal scale to dimension at bottom, which is former diameter of fringe $N-2$. Hence, fraction indicated by fringe $N-1$ is identical to fractional portion of central order.

the image of a fringe. Measurement is made using the fringe which appears within the scale limits and the indicated subdivision upon which it falls. This value is the required fractional order at the pattern center, and hence the fractional half-wavelength portion of the plate separation. Measurements are made to an accuracy of 0.05 fringes $\left(1.36 \times 10^{-5}\right.$ millimeters $)$ which, for a 25 meter-long instrument, represents a strain sensitivity of $5.6 \times 10^{-10}$.

Figure 10 is a composite photograph showing the correlation between an eightday sequence of interferometer exposures from June 12 to June 19, 1964 (Figure 7) and the corresponding analog record. One particular order of the green line, designated $b$, is easily identified by the coincidence of green, blue, and yellow, and can be followed throughout the sequence. The analog record time scale has been compressed and the record inverted (dilatation upward) to demonstrate the corres- 
pondence with fringe movement. Small circles on the trace indicate the times at which the daily exposures were made, and the order of interference for each of these points, measured from the fringe pattern, are tabulated below:

$\begin{array}{cccccccc}\text { June } 12 & 13 & 14 & 15 & 16 & 17 & 18 & 19 \\ b+6.5 & b+7.7 & b+9.6 & b+10.6 & b+11.2 & b+13.1 & b+13.5 & b+12.9\end{array}$

Correlation is good to within two-tenths of a fringe up to June 17. However, a pronounced divergence of the ink trace in the direction of dilatation beginning June

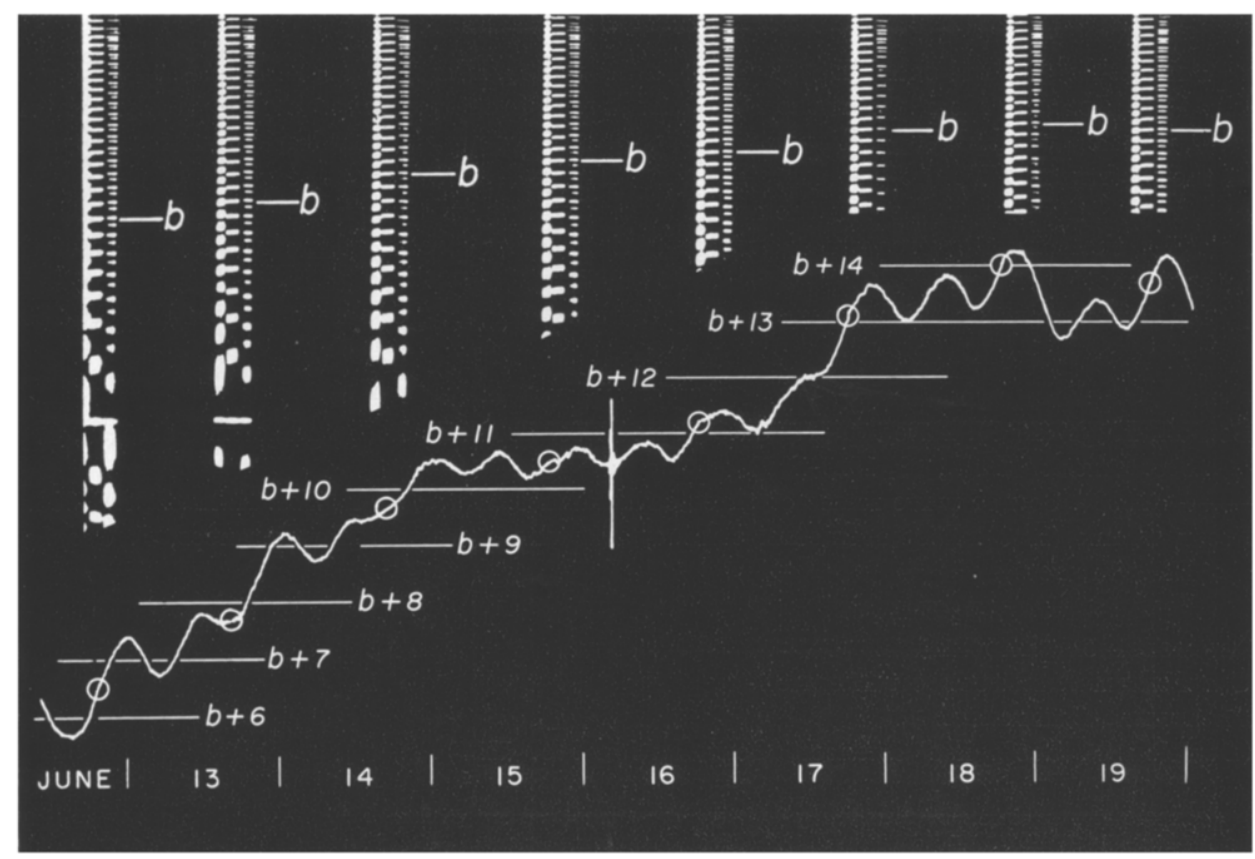

Fig. 10. Comparison of interferometric photograph and corresponding analog record. Small circles indicate times of exposures. Vertical scale was constructed from interferometric data, providing accurate calibration of analog record. One division equals one-half wavelength of mercury green line $\left(0.273 \times 10^{-3}\right.$ millimeters $)$ or $1.12 \times 10^{-8}$ strain.

18, and a corresponding deterioration in the sharpness of the fringes, which became quite noticeable a few days later, seemed to indicate a lateral shifting of the quartz. (Shifting of the quartz would produce skewness in the interferometer plates and a false axial motion of the capacity-sensing plate which is not on the quartz axis.) This was confirmed by the return to normal of the fringe definition when a lateral readjustment of the quartz was made, which shifted the recorder trace in a direction to reduce the divergence. Over the months of operation these divergences have been gradually decreasing, indicating an eventual settling of disturbing forces in the piers and quartz tube mounts. Concern over these discrepancies, however, is somewhat unwarranted since at no time have the excursions of the parallelism of the inter- 
ferometer plates even approached the magnitude of one fringe, or a strain figure of $10^{-8}$.

\section{ConCLUSion}

The strain meter described has been in continuous operation since February 29, 1964 with no down-time except during occasional power failures. Recovery of the reference zero is available at any time by re-examination of the back-dated interferometer photographs. Recent work by Press (1965) provides an example of combined use of the interferometric and continuous ink records.

\section{ACKNOWLEDGMENTS}

The authors wish to thank Lt. Commander Robert C. Munson, Mr. Robert A. Eppley, and Mr. Ron Viets, all members of the U. S. Coast and Geodetic Survey Honolulu Observatory staff, for their valuable suggestions and assistance relative to the installation and maintenance of the instrument. We are also grateful to Dr. Frank Press for his supervision of the project and, in addition, for his help in the actual assembly. Special thanks are due Mr. J. M. Nordquist for the computer program and mathematical analysis required in the preparation of the interferometric tables.

This research was partially supported by National Science Foundation Grant No. GP-2806.

\section{REFERENCES}

Benioff, Hugo (1959). Fused quartz extensometer for secular, tidal, and seismic strains, Geol. Soc. America Bull., 70, 1019-1032.

(1963). Source wave forms of three earthquakes, Bull. Seism. Soc. Am., 53, 893-903.

Press, Frank (1965). Displacements, strains, and tilts at teleseismic distances, J. Geophys. Res. (in press).

Seismological Laboratory

California Institute of Technologx

Pasadena, Califoria

(Division of Geological Sciences, Contribution No. 1317)

Manuscript received February 18, 1965. 